\title{
Late-onset Hereditary Transthyretin Amyloidosis in Two Patients with Acquired Demyelinating Features
}

\author{
Nazila Rad and Said R Beydoun \\ Neuromuscular Division, Department of Neurology, Keck School of Medicine, University of Southern California, Los Angeles, CA, US
}

A utosomal-dominant transthyretin (TTR)-related amyloidosis usually manifests in the third to fifth decade with a length-dependent axonal neuropathy and prominent involvement of the small diameter nerve fibers. Objectives: To describe the clinical and para-clinical findings in patients with hereditary transthyretin amyloidosis (hATTR), formerly known as transthyretin-related familial amyloid polyneuropathy (TTR-FAP). Methods: Electrodiagnostic, cerebrospinal fluid (CSF), and TTR gene findings in two patients misdiagnosed with chronic inflammatory demyelinating polyneuropathy (CIDP). Results: A 78-year-old, right-handed, Caucasian male (patient 1) and a 69-year-old, right-handed, Japanese male (patient 2) were referred for late-onset sensory symptoms of the hands and feet as initial manifestations. The first patient, after several years, developed progressive leg weakness affecting his gait and balance, as well as dysautonomic complaints. The second patient had relatively rapid progression with bilateral foot drop and ambulation difficulty after a few months. In both patients, CSF findings were unremarkable. Lumbar spine magnetic resonance imaging did not reveal abnormal thickening or enhancement of the lumbar plexus and exiting nerve roots. Both patients were initially diagnosed with CIDP before being referred to our institution. Patient 2 was started on intravenous immunoglobulin by his primary neurologist, which was maintained for a year without a meaningful response. Repeat electrodiagnostic study at our institution revealed non-length-dependent axonal sensory loss and features of acquired demyelinating neuropathy. TTR gene testing identified pathogenic variants p.Val30Met or V30M, and p.Ala 117Ser or A117S, in the first and the second patient, respectively. Conclusion: hATTR can mimic CIDP clinically and electrodiagnostically. The presence of significant sensory axonal loss, rapid course, and lack of response to immunomodulation therapy should prompt consideration of this diagnosis and TTR gene testing.

\section{Keywords}

Hereditary transthyretin amyloidosis (hATTR), transthyretin-related familial amyloidosis polyneuropathy (TTR-FAP), chronic inflammatory demyelinating polyneuropathy (CIDP), TTR gene, progressive neuropathy, antisense oligonucleotides, RNAi

Disclosures: Nazila Rad has nothing to disclose in relation to this article. Said R Beydoun serves on an advisory board, as a consultant or speaker for Alnylam Pharmaceuticals and Akcea Therapeutics.; he is also the journal's Editor-in-Chief.

Review Process: Double-blind peer review.

Compliance with Ethics: Procedures were followed in accordance with the responsible committee on human experimentation and with the Helsinki Declaration of 1975 and subsequent revisions. Informed consent was received from both patients for publication of this case report.

Authorship: The named authors meet the International Committee of Medical Journal Editors (ICMJE) criteria for authorship of this manuscript, take responsibility for the integrity of the work as a whole, and have given final approval for the version to be published.

open Access: This article is published under the Creative Commons Attribution Non-commercial License, which permits any non-commercial use, distribution, adaptation, and reproduction provided the original authors and source are given appropriate credit. (C) The Authors 2018.

Received: June 4, 2018

Accepted: August 28, 2018

Citation: US Neurology. 2018;14(2):98-101

Corresponding Author: Said R Beydoun, Leslie P Weiner Neurological Research Institute, Keck School of Medicine, University of Southern California, 1520 San

Pablo Street, Suite 3000, Los Angeles, CA 90033, US. E: sbeydoun@usc.edu

Support: No funding was received in the publication of this article.
Hereditary transthyretin amyloidosis (hATTR), formerly known as transthyretin-related familial amyloid polyneuropathy (TTR-FAP), is the most common form of genetic amyloidosis, transmitted as an autosomal dominant trait. $\mathrm{V} 30 \mathrm{M}$ is the most common identified mutation in patients with polyneuropathy, with endemic foci in Portugal, northern Sweden, and Japan. However, more than 100 other mutations have been described, with varying geographic distributions and organ involvement. The most typical presentation of hATTR is a progressive length-dependent sensory-motor polyneuropathy, which usually begins with small fiber involvement in patients with a history, or presence, of carpal tunnel syndrome. ${ }^{1}$

Diagnosis is based on the clinical history, presence of other systemic complaints, family history (which can be lacking because of incomplete and variable penetrance), electrodiagnostic evidence of axonal polyneuropathy, identification of amyloid deposits in the tissues, and detection of TTR mutation. The diagnosis can be challenging in sporadic cases and when the clinical manifestations are atypical. Diagnostic pitfalls include inadequate attention to the presence of autonomic symptoms, as well as the presence of demyelinating features on nerve conduction study, leading to an erroneous diagnosis of chronic inflammatory demyelinating polyneuropathy (CIDP). ${ }^{2,3}$

Here we present a retrospective analysis of two patients, seen within a 6-12-month period, at our neuromuscular division. The patients were initially diagnosed with CIDP. An hATTR diagnosis was made on the basis of the presence of neuropathy in conjunction with two different pathogenic mutations in the TTR gene. 


\section{Case presentation Patient 1}

A 78-year-old, right-handed, Caucasian male was referred for evaluation of progressive sensory motor polyneuropathy. He developed numbness in his feet 7 years prior to his visit, which gradually progressed to burning pain of the feet and legs, ascending to his knees, and then involving his hands. Later, he developed foot drop affecting his gait, causing frequent falls. Autonomic symptom inventory included constipation, change in his rate of sweating, orthostatic dizziness, postprandial fullness, and nausea.

Pertinent findings on neurologic examination revealed symmetric distal muscle weakness in the distal upper and lower extremities. Muscle testing by Medical Research Council (MRC) scale of $0-5$ revealed particular weakness in the ankle dorsiflexors (3+), ankle plantarflexors (4), thenars (3+), and interossei (3+). He had absent vibration sensation at the toes and the malleoli with loss of pinprick to the midcalf, absent proprioception at the toes, absent reflexes, and a steppage gait.

Diagnostic work-up included: glycated hemoglobin (HDA1C), thyroid-stimulating hormone (TSH) levels, vitamin B12, homocysteine, methylmalonic, immunofixation, cytoplasmic anti-neutrophil cytoplasmic antibodies (c-ANCA), perinuclear-ANCA ( $\mathrm{p}$-ANCA), and cryoglobulins. All tests revealed negative or normal levels. Paraneoplastic panel, including anti-Hu antibody, was negative. Cerebrospinal fluid (CSF) examination revealed protein of $52 \mathrm{mg} / \mathrm{dL}$. Prior to referral at our institution, the patient had been diagnosed with CIDP.

Repeat electrodiagnostic study at our institution revealed non-length-dependent axonal sensory motor polyneuropathy with prolonged distal latencies and decreased conduction velocity in both the left median and left peroneal nerves, with no conduction block (Table 1). The patient did not have any cardiac symptoms, and cardiac evaluation including an echocardiogram showed left ventricular hypertrophy with an ejection fraction of 55-60\%. TTR gene testing identified pathogenic variants (p.Val30Met). There was no known family history of neuropathy.

\section{Patient 2}

A 69-year-old, right-handed, Japanese male was referred for evaluation of progressive weakness and ambulation difficulty. His symptoms began with progressive acral numbness followed by bilateral foot drop over the course of a year. The weakness progressed significantly to the point that he became wheelchair-bound a year later. Autonomic complaints included constipation and postprandial fullness. He had prior diagnosis of bilateral carpal tunnel and decompressive surgery several years earlier.

Pertinent findings on neurologic examination revealed more distal than proximal weakness in bilateral upper extremities right more than left, as well as symmetric distal weakness in lower extremities. Muscle testing by MRC scale of $0-5$ revealed: deltoids (4+), triceps and biceps (5-), interossei (4), thenar (3-), hip flexors (5-), ankle dorsiflexion (2-) on the right, (0) on the left. He had pseudoathetoid movement in the fingers with significant loss of proprioception, vibration, and light touch in both lower and upper extremities.

Diagnostic work-up included the following: HbA1C, TSH, vitamin B12, homocysteine, methyl malonic acid, immunofixation, C-ANCA, and p-ANCA. All tests revealed negative or normal levels. The paraneoplastic
Table 1: Electrodiagnostic findings of selected nerves in patient 1

\begin{tabular}{|c|c|c|c|c|c|}
\hline Nerve/sites & Muscle & $\begin{array}{l}\text { Latency } \\
\text { ms }\end{array}$ & $\begin{array}{l}\text { Amplitude } \\
\mathrm{mV}\end{array}$ & $\begin{array}{l}\text { Velocity } \\
\mathrm{m} / \mathrm{s}\end{array}$ & $\begin{array}{l}\text { Area } \\
\%\end{array}$ \\
\hline L-peroneal & Tib ant & & & & \\
\hline Fib head & Tib ant & 10.7 & 0.4 & \multirow{3}{*}{$\begin{array}{l}22 \\
\geq 44\end{array}$} & 100 \\
\hline Normative values & & $<6$ & $\geq 5$ & & \\
\hline Pop fossa & Tib ant & 13.4 & 0.3 & & 76.3 \\
\hline L-median & \multicolumn{5}{|l|}{ APB } \\
\hline Wrist & APB & 5.7 & 2.8 & \multirow{3}{*}{$\begin{array}{l}47 \\
\geq 49\end{array}$} & 100 \\
\hline Normative values & & $<4.4$ & $\geq 5$ & & \\
\hline Elbow & APB & 10.8 & 2.6 & & 83.6 \\
\hline
\end{tabular}

\begin{tabular}{|l|l|l|l|l|l|}
\hline Nerve/sites & Rec. site & $\begin{array}{l}\text { Peak latency } \\
\mathrm{ms}\end{array}$ & $\begin{array}{l}\text { Amplitude } \\
\mathrm{mV}\end{array}$ & $\begin{array}{l}\text { Velocity } \\
\mathrm{m} / \mathrm{s}\end{array}$ \\
\hline L-sural & Ankle & $\mathrm{NR}$ & $\mathrm{NR}$ & $\mathrm{NR}$ \\
\hline & & $<4.3$ & $>6$ & $>40$ \\
\hline L-median & Dig II & $\mathrm{NR}$ & $\mathrm{NR}$ & $\mathrm{NR}$ \\
\hline & & $\leq 3.7$ & $\geq 20$ & $\geq 50$ \\
\hline
\end{tabular}

$A P B=$ abductor pollicis brevis; Dig $\|=$ digit $\|$; Fib = fibular head; $L=$ left; $m s=$ millisecond; $m V=$ millivolt; $\mathrm{m} / \mathrm{s}=$ meters per second; $N R=$ not recorded; $R e c .=$ recording; Tib ant $=$ tibialis anterior.

antibody panel was negative. CSF examination revealed normal cell count and protein. The patient was diagnosed with CIDP and was started on intravenous immunoglobulin (IVIG) by his primary neurologist and maintained for a year. He was subsequently referred to our institution for re-evaluation, given no effective response to immunomodulatory therapy.

Repeat electrodiagnostic study at our institution revealed non-length-dependent axonal sensory motor polyneuropathy with features of acquired demyelinating neuropathy in bilateral median nerves with significant conduction block and slow conduction velocity in the right median nerve. Demyelinating changes were noted in the ulnar sensory nerves (Table 2).

The patient did not have any cardiac symptoms. Cardiac evaluation including heart magnetic resonance imaging (MRI) with contrast revealed moderate asymmetric hypertrophy of the left ventricular basal to mid septal wall, with no outflow tract obstruction. Calculated left ventricular ejection fraction was 61\%. Fourteen days of heart monitoring was unremarkable. Lumbar spine MRI did not reveal abnormal thickening or enhancement of the lumbar plexus and existing nerve roots. TTR gene testing identified pathogenic variants (p.Ala 117Ser). There was no family history of neuropathy.

A summary of findings in patients 1 and 2 is shown in Table 3.

\section{Discussion}

hATTR, or Corino de Andrade's disease, ${ }^{1}$ was first identified and described by a Portuguese neurologist, Mario Corino da Costa Andrade, in 1952. ${ }^{2}$ hATTR is transmitted as an autosomal dominant trait arising from mutations of the TTR gene with variable penetrance. ${ }^{3}$ Penetrance is significantly higher with a risk of earlier onset when the mutation is inherited from the mother rather than from the father. ${ }^{4}$ hATTR is caused by any one of more than 100 mutations in the TTR gene. 
Table 2: Electrodiagnostic findings of selected nerves in patient 2

\begin{tabular}{|l|l|l|l|l|l|l|}
\hline Nerve/sites & Muscle & $\begin{array}{l}\text { Latency } \\
\mathrm{ms}\end{array}$ & $\begin{array}{l}\text { Amplitude } \\
\mathrm{mV}\end{array}$ & $\begin{array}{l}\text { Velocity } \\
\mathrm{m} / \mathrm{s}\end{array}$ & $\begin{array}{l}\text { Area } \\
\%\end{array}$ \\
\hline R-Median & APB & \multicolumn{2}{|l|}{} & & \\
\hline Wrist & APB & 3.5 & 3.4 & 16 & 100 \\
\hline Normative values & & $<4.4$ & $\geq 5$ & $>49$ & \\
\hline Elbow & APB & 16.8 & 0.2 & & 5.07 \\
\hline L-median & APB & & & & \\
\hline Wrist & APB & 4.5 & 2.2 & not calculable & 100 \\
\hline Normative values & & $<4.4$ & $\geq 5$ & $\geq 49$ & \\
\hline Elbow & APB & 10.3 & 0.2 & & 16.2 \\
\hline
\end{tabular}

\begin{tabular}{|l|l|l|l|l|}
\hline & Rec. site & $\begin{array}{l}\text { Peak latency } \\
\text { ms }\end{array}$ & $\begin{array}{l}\text { Amplitude } \\
\mathrm{mV}\end{array}$ & $\begin{array}{l}\text { Velocity } \\
\mathrm{m} / \mathrm{s}\end{array}$ \\
\hline R-sural & Ankle & $\mathrm{NR}$ & $\mathrm{NR}$ & $\mathrm{NR}$ \\
\hline & & $<4.3$ & $\geq 6$ & $\geq 40$ \\
\hline R-median & Dig II & $\mathrm{NR}$ & $\mathrm{NR}$ & $\mathrm{NR}$ \\
\hline & & $\leq 3.7$ & $\geq 20$ & $\geq 50$ \\
\hline R-ulnar & Dig V & 7.6 & 5.4 & 22 \\
\hline & & $<3.7$ & $>17$ & $>50$ \\
\hline
\end{tabular}

$A P B=$ abductor pollicis brevis; Dig $\|=$ digit Il; Dig V = digit $\mathrm{V} ; \mathrm{L}=$ left; $m s=$ millisecond; $m V=$ millivolt; $m / s=$ meters per second; $N R=$ not recorded; $R=$ right; $R e c .=$ recording .

Table 3: Summary of the findings in patient 1 and patient 2

\begin{tabular}{|l|l|l|}
\hline Age at onset & Patient 1 & Patient 2 \\
\hline Gender & 70 & 67 \\
\hline Ethnicity & Male & Male \\
\hline Sensory symptoms & Caucasian & Japanese \\
\hline Autonomic symptoms & Present at onset & Present at onset \\
\hline Progression interval & Present & Present \\
\hline CSF protein (mg\%) & $7-8$ years & 3 years \\
\hline Mutation & 52 & 45 \\
\hline & V30M & A117S \\
\hline
\end{tabular}

CSF $=$ cerebrospinal fluid.

TTR is synthesized by the liver (95\%), the choroid plexus of the brain, and the retinal pigment epithelium. The TTR protein is a tetramer that transports thyroxine and retinol binding protein. The tetramer has to dissociate into misfolded monomers to aggregate into a variety of structures including amyloid fibrils. Single substitutions in an amino acid of TTR can render it unstable, resulting in a tendency to misfold and produce amyloid. This protein is encoded by a small gene containing four exons, located on the long arm of chromosome $18 .^{5}$ These fibrils infiltrate the peripheral and autonomic nerves or myocardium and cardiac conduction system, and can also build up in other organs (e.g., gastrointestinal tract, kidneys, and eyes). ${ }^{1,5}$ They disrupt normal tissue structure and lead to progressive organ failure. ${ }^{1,5}$ Phenotypic variations depend upon their type, location, and the amount of deposition; which makes it difficult to recognize across individuals and geographic locations. Certain mutations produce a primarily neurological phenotype (e.g., V30M'), while others are associated almost exclusively with cardiac problems (e.g., V12216.7), whereas numerous other mutations induce mixed phenotypes.

Patient 1 was a Caucasian male with late-onset sensorimotor polyneuropathy affecting his balance over a relatively rapid course of 8 years. He had a V30M mutation; this particular mutation is mostly identified in patients aged from 32-35 years in endemic areas of Brazil, Portugal, and Japan, to 56.7 and 61 years in Sweden and Majorca, respectively. In the first report of the disease, the duration of the disease among Portuguese patients ranged from 7-10 years. ${ }^{9}$ Subsequent reports showed in endemic regions (Portugal, Cyprus, and Majorca), that hATTR often occurs before the age of 40 (early onset), with progressive sensory-motor and autonomic neuropathy, leading to cachexia and eventually death within, on average, 10.8 years of disease onset..$^{10}$

Patient 2 was a Japanese male with late-onset progressive motor weakness observed over a more rapid course of 3 years." $\mathrm{He}$ had the variant A117S; this mutation replaces alanine with serine at codon 117 of the TTR protein, and has been described in the Taiwanese population. A hospital-based study in Taipei, Taiwan suggests that A117S may be a significant genetic etiology for chronic pan-modality polyneuropathy of adult onset. In this study, 19 patients with this mutation exhibited clinical and electrophysiologic evidence of idiopathic adult-onset axonal polyneuropathy. ${ }^{12}$ Almost all A117S patients had a late age at onset (>50 years of age), unlike V30M patients who can have both early- and late-onset types, ${ }^{13}$ as described above. ${ }^{8}$

In both patients, the electrodiagnostic studies showed non-length-dependent sensorimotor axonal neuropathy with features of acquired demyelination, which could have been easily missed due to the presence of significant axonal findings. The latter could also have been due to the chronicity and progression of the neuropathy. Given the normal CSF protein levels and the normal MRI of nerve roots, as well as no response to IVIG in patient 2, the diagnosis of CIDP appeared to be questionable in both patients. The presence of gastrointestinal autonomic symptoms in both patients, as well as a history of bilateral carpal tunnel syndrome in patient 2, prompted us to consider gene testing for hATTR. Patient 1 had V30M mutation which has now been reported worldwide. Patient 2 had A117S mutation, which is a rare mutation that, until now, has never been described in the Japanese population.

One of the major ongoing challenges in hATTR is to identify the early, and sensitive, biomarkers in order for earlier initiation of therapies, and to enable clinical trials in early-stage patients, before irreversible axonal neural degeneration has occurred. Electrophysiologic studies are important parts of the diagnostic evaluation. These may include nerve conduction studies, electromyography, and autonomic function study including quantitative sudomotor axon reflex testing (QSART). Electromyography and nerve conduction studies are useful in demonstrating large fiber neuropathy in sufficiently advanced cases, whereas studies such as autonomic function study and QSART are useful in demonstrating small fiber involvement, which is typically involved in the early phase of the disease. Nerve biopsy may be considered for cases in which the diagnosis remains elusive after less invasive tests have been inconclusive. Nerve biopsies in patients with hATTR reveal amyloid deposits in the endoneurial and epineurial connective tissue, along with deposits in the endoneurial and epineurial blood-vessel walls. With congo red staining, amyloid exhibits an 
apple-green birefringence under polarized light and appears red when viewed under a light microscope. ${ }^{14,15}$ Amyloid deposits may also be seen in biopsy specimens of muscle, abdominal fat, or salivary glands. ${ }^{1}$

For years, liver transplantation was the only available treatment to prevent the formation of additional amyloid deposits by removing the main source of mutated TTR. If liver transplantation is performed early in the course of hATTR, it may increase the median survival to more than 20 years. ${ }^{16}$

There are few available pharmacologic therapies worldwide. TTR stabilizers (diflunisal and tafamidis) are pharmacologic therapies of hATTR which target the rate-limiting step in the formation of amyloid fibrils. ${ }^{17}$ Diflunisal, a generic nonsteroidal anti-inflammatory drug, can reduce the rate of progression of polyneuropathy and preserve quality of life in patients with hATTR; ${ }^{18}$ however, diflunisal has limitations in participants with cardiomyopathy or heart failure due to its sodium retention side effects.

Tafamidis meglumine is approved by the European Union for the treatment of hATTR; it binds to the two thyroxine-binding sites on the native tetrameric form of TTR and prevents dissociation into monomers and formation of amyloid. A randomized double-blind placebo-controlled trial showed a disease-modifying effect on neuropathy, neurophysiological function, and especially on quality of life and body mass index. ${ }^{19}$

There are several emerging pharmacologic therapies designed to slow or stop the progression of hATTR. Hence, it is imperative to diagnose this condition accurately especially given the emergence of novel potential pharmacotherapy agents such as RNA interference (RNAi) and antisense oligonucleotides (ASO). These agents selectively silence TTR gene expression both in vitro and in vivo. ${ }^{20,21}$ Data demonstrate that the long-term administration of patisiran (RNAi) and inotersen (ASO) are generally well-tolerated. Phase III clinical trials met the primary efficacy end-point, showing slowing of the neuropathy progression and improvement in the patients' quality of life. Results have also shown robust and sustained serum TTR lowering, supporting the therapeutic hypothesis that TTR knockdown can potentially prevent and reverse the neuropathy. ${ }^{22-24}$

In conclusion, hATTR is a progressive and fatal disease that is increasingly diagnosed worldwide. Neurologists must be aware of the diagnostic pitfalls of hATTR, and gene sequencing should be done in all suspected cases. The importance of correct diagnosis is crucial in order to avoid unnecessary therapy, such as immune therapy (as in our second patient, receiving IVIG for 1 year) while the disease is progressing. More importantly, the potential future availability of disease-modifying therapies that target the mutant gene adds more impetus to the avoidance of misdiagnosis and the identification of disease characteristics. Genetic testing should be also encouraged in relatives of diagnosed cases when they are able to understand its medical, social, and psychological consequences. A good knowledge of the natural history of the disease according to different TTR mutations allows clinicians to optimize multidisciplinary care for patients and to offer carriers a personalized follow-up to reveal the first signs of the disease. $\square$
1. Planté-Bordeneuve $\mathrm{V}$, Said G. Familial amyloid polyneuropathy Lancet Neurol. 2011;10:1086-97.

2. Planté-Bordeneuve V, Ferreira A, Lalu T, et al. Diagnostic pitfalls in sporadic transthyretin familial amyloid polyneuropathy (TTR-FAP). Neurology. 2007:69:693-8.

3. Cappellari $\mathrm{M}$, Cavallaro T, Ferrarini $\mathrm{M}$, et al. Variable presentations of TTR-related familial amyloid polyneuropathy in seventeen patients. J Peripher Nerv Syst. 2011;16:119-29.

4. Ando $Y$, Ueda M. Novel methods for detecting amyloidogenic proteins in transthyretin related amyloidosis. Front Biosci. 2008;13:5548-58

5. Merlini G, Bellotti V. Molecular mechanisms of amyloidosis. N Engl IMed. 2003;349:583-96.

6. Mankad AK, Shah KB. Transthyretin cardiac amyloidosis. Curr Cardiol Rep. 2017:19:97.

7. Connors LH, Prokaeva T, Lim A, et al. Cardiac amyloidosis in African-Americans: comparison of clinical and laboratory features of transthyretin V122I amyloidosis and immunoglobulin light chain amyloidosis. Am Heart J. 2009;158:607-14.

8. Hellman $\mathrm{U}$, Alarcon F, Lundgren $\mathrm{HE}$, et al. Heterogeneity of penetrance in familial amyloid polyneuropathy, ATTR Val30Met, in the Swedish population. Amyloid 2008:15:181-6.

9. Reinés JB, Vera TR, Martín MU, et al. Epidemiology of transthyretin-associated familial amyloid polyneuropathy in the
Majorcan area: Son Llàtzer Hospital descriptive study. Orphanet $\lrcorner$ Rare Dis. 2014;9:29

10. Koike H, Misu K, Sugiura M, et al. Pathology of early- vs late-onset TRR Met30 familial amyloid polyneuropathy. Neurology. 2004:63:129-38.

11. Yang, NC, Lee MJ, Chao CC, et al. Clinical presentations and skin denervation in amyloid neuropathy due to transthyretin Ala97Ser. Neurology. 2010;75:532-8

12. Coutinho P, A Lázaro da Silva, J Lopes, et al. Forty years of experience with type 1 amyloid neuropathy: review of 483 cases. In: Glenner GG, Costa PP, Freitas F, eds. Amyloid and Amyloidosis. Amsterdam, the Netherlands: Excerpta Medica. 1980;88-98.

13. Adams D, Samuel D, Goulon-Goeau C, et al. The course and prognostic factors of familial amyloid polyneuropathy after liver transplantation. Brain. 2000:123:1495-504.

14. Simmons Z, Specht C. The neuromuscular manifestations of amyloidosis. J Clin Neuromusc Dis. 2010;11:145-57.

15. Li K, Kyle RA, Dyck PJ. Immunohistochemical characterization of amyloid proteins in sural nerves and clinical associations in amyloid neuropathy. Am J Pathol. 1992;141:217-26.

16. Okamoto S, Wixner J, Obayashi K, et al. Liver transplantation for familial amyloidotic polyneuropathy: impact on Swedish patients survival. Liver Transpl. 2009;15:1229-35.

17. Hou X, Aguilar MI, Small DH. Transthyretin and familial amyloidotic polyneuropathy. Recent progress in understanding the molecular mechanism of neurodegeneration. FEBS J. 2007;274:1637-50.

18. Berk JL, Dyck PJ. Repurposing diflunisal for familial amyloid polyneuropathy: a randomized clinical trial. JAMA. 2013:310:2658-67.

19. Coelho T, Maia L, Martins da Silva A, et al. Tafamidis (FX-1006A): a first-in-class disease-modifying therapy for transthyretin familial amyloid polyneuropathy. Neurology. 2010;74:A286.

20. Kurosawa T, Igarashi S, Nishizawa M, Onodera O. Selective silencing of a mutant transthyretin allele by small interfering RNAS. Biochem Biophys Res Commun. 2005;337:1012-8.

21. Freeman $R$, Barroso F. Recent advances in familial amyloid polyneuropathy. Curr Opin Neurol. 2015;28:494-9.

22. Butler JS, Chan A, Costelha S, et al. Preclinical evaluation of RNAi as a treatment for transthyretin-mediated amyloidosis. Amyloid. 2016:23:109-18

23. Adams $D$, Coelho T, Conceicao I, et al. Phase 2 open-label extension (OLE) study of patisiran, an investigational RNA interference (RNAi) therapeutic for the treatment of hereditary ATTR amyloidosis with polyneuropathy. Neurology. 2017:88(16 Suppl):S27.004

24. Hawkins PN, Ando Y, Dispenzeri A, et al. Evolving landscape in the management of transthyretin amyloidosis. Ann Med. 2015;47:625-38 